\title{
The Radio Frequency Environment at 240-270 MHz with Application to Signal-of-opportunity Remote Sensing
}

Jeffrey R. Piepmeier ${ }^{1}$, Manuel Vega ${ }^{1}$, Matthew Fritts ${ }^{1,2}$, Cornelius DuToit ${ }^{1,3}$, Joseph Knuble ${ }^{1}$, Yao-Cheng Lin ${ }^{4}$, Benjamin Nold ${ }^{4}$, James Garrison ${ }^{4}$

\section{IGARSS 2017 \\ Fort Worth, TX}

${ }^{1}$ NASA Goddard Space Flight Center, Greenbelt, MD, USA

${ }^{2}$ SGT, Inc., Greenbelt, MD 20771, USA ${ }^{3}$ AS\&D, Inc., Greenbelt, MD 20771, USA

${ }^{4}$ Purdue University, West Lafayette, IN, USA 


\section{Outline}

- P-band, Soil Moisture, and SoOp

- SoOp-AD and Results

- RFI Effects in SoOp

- Spectrum Observations

- Conclusions

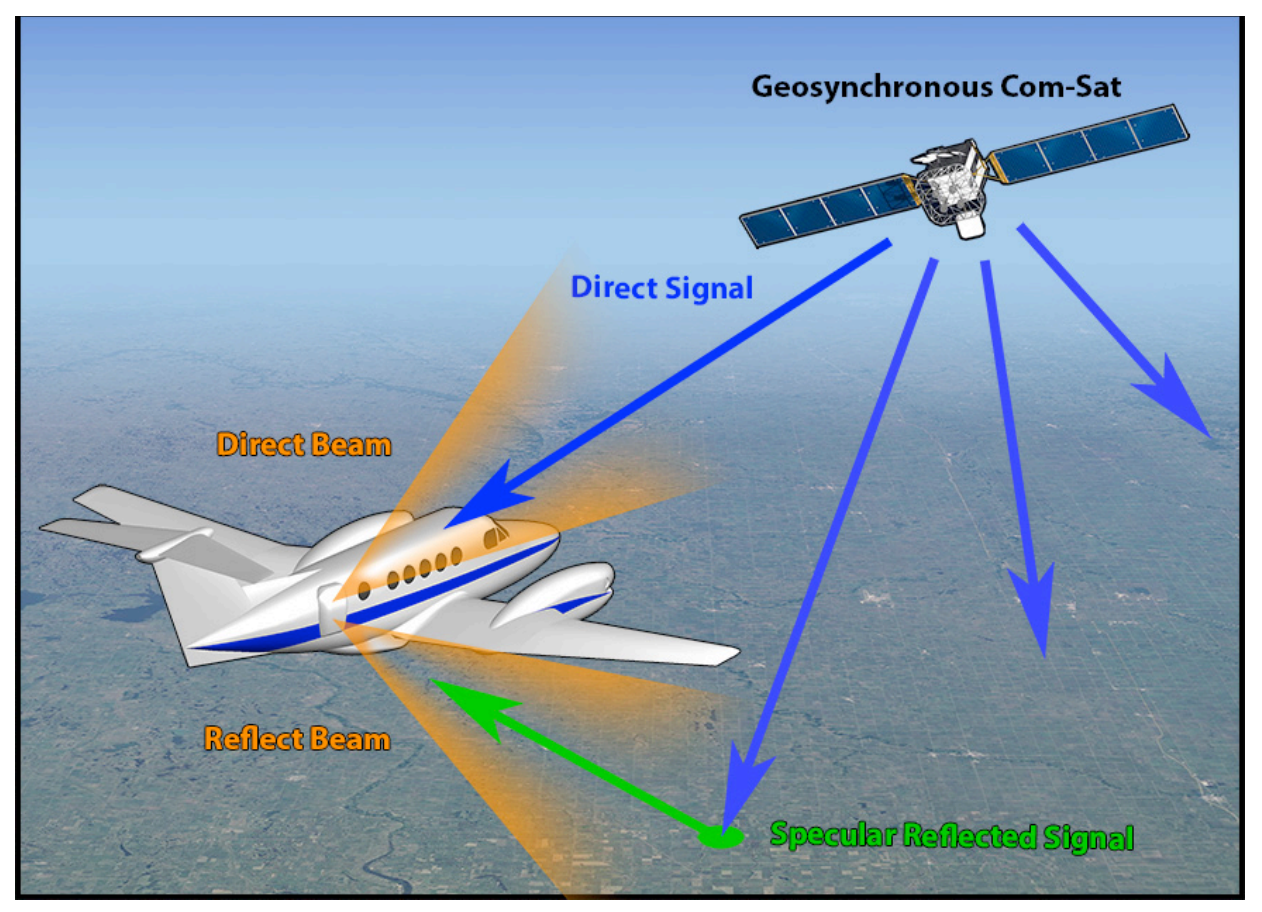




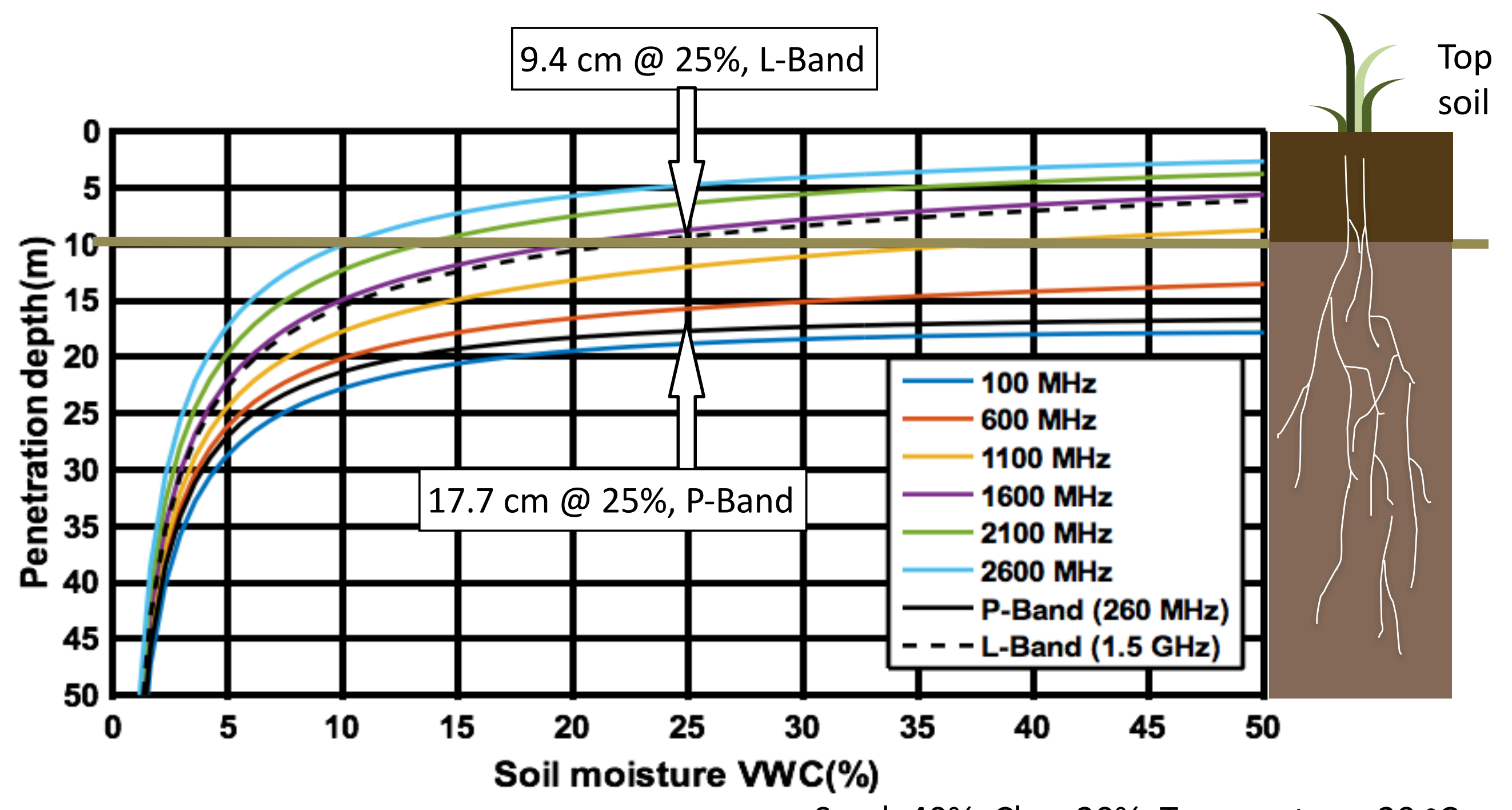

Sand: $40 \%$, Clay: $20 \%$, Temperature: $20^{\circ} \mathrm{C}$ 


\section{Soil P-band Reflectivity}

\section{Assumption:}

1. Flat specular reflection

2. Single layer and media

3. No vegetation effect

Specular reflection

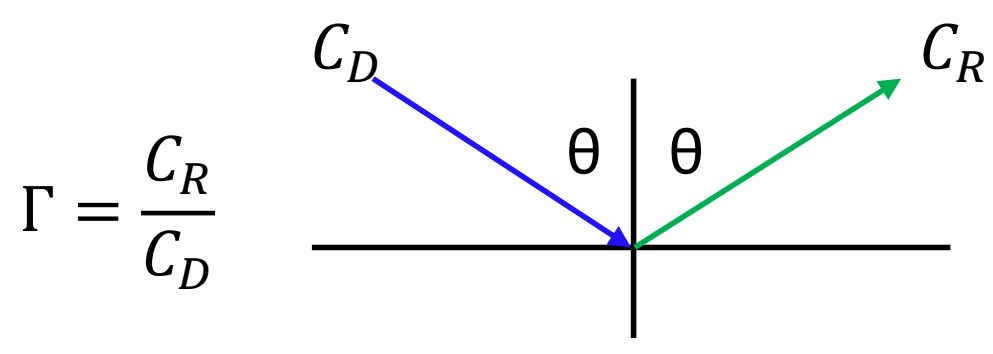

$\Gamma$ : Reflectivity

$C_{D}$ : Carrier power for direct signal

$C_{R}$ : Carrier power for reflected signal
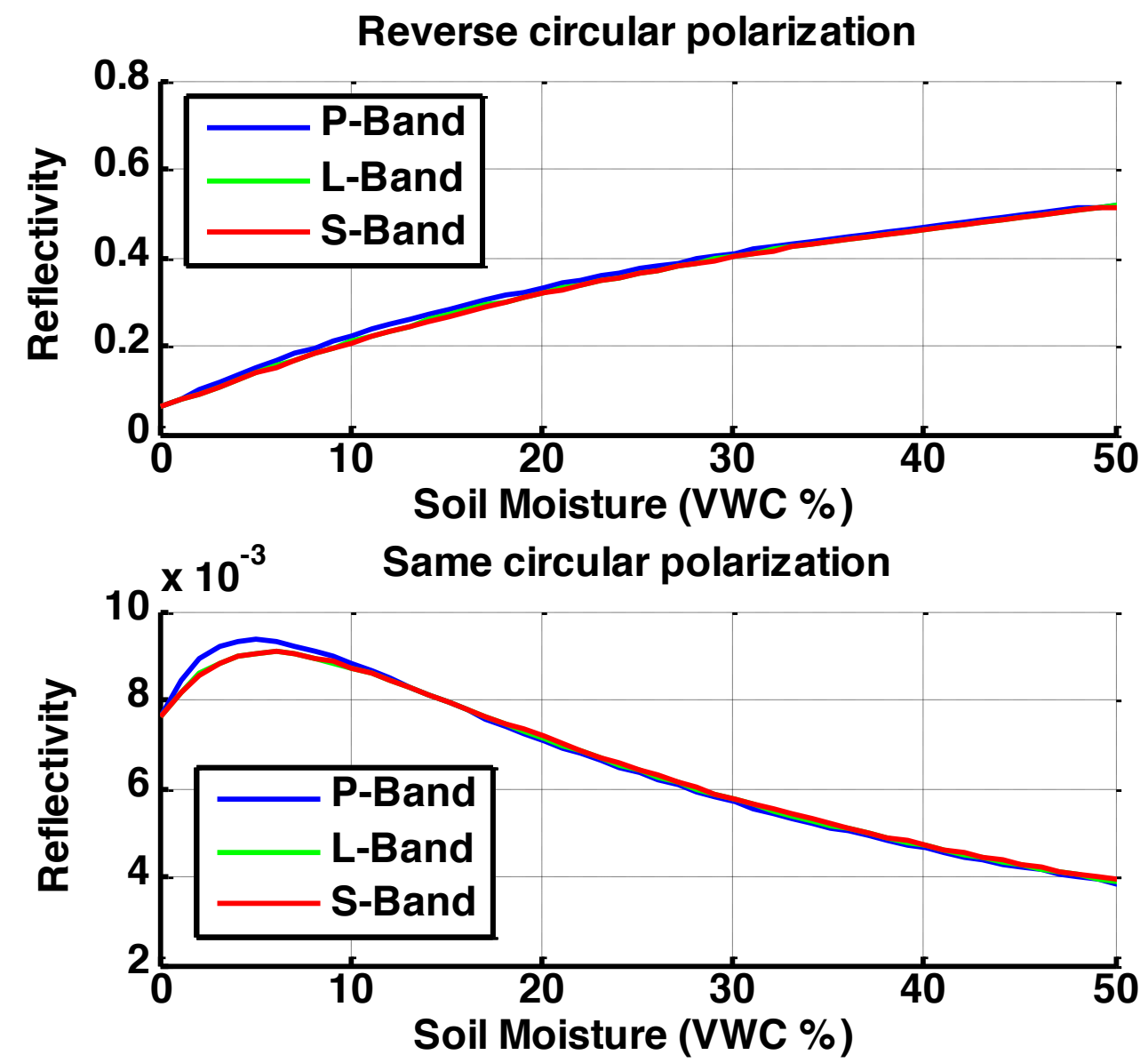


\section{P-band Signals of Opportunity (SoOp)}

- Re-utilization of existing transmissions (e.g. potential RFI sources)

- No transmit permission required (re: ESA's Biomass)

- Bands allocated for Space-Earth communications

- High power, forward scatter -> High SNR/smaller antenna

- Resolution set by signal bandwidth or Fresnel zone

\section{P-band SoOp offers opportunity to measure deeper soil moisture from space at low cost}




\section{(10.8 Grodand}

\section{Sources for P-band SoOp}

- 225-420 MHz allocation for defense/government use

- Continuous use by US \& Others since 1978 (FLTSATCOM)

- Planned utilization through 2024

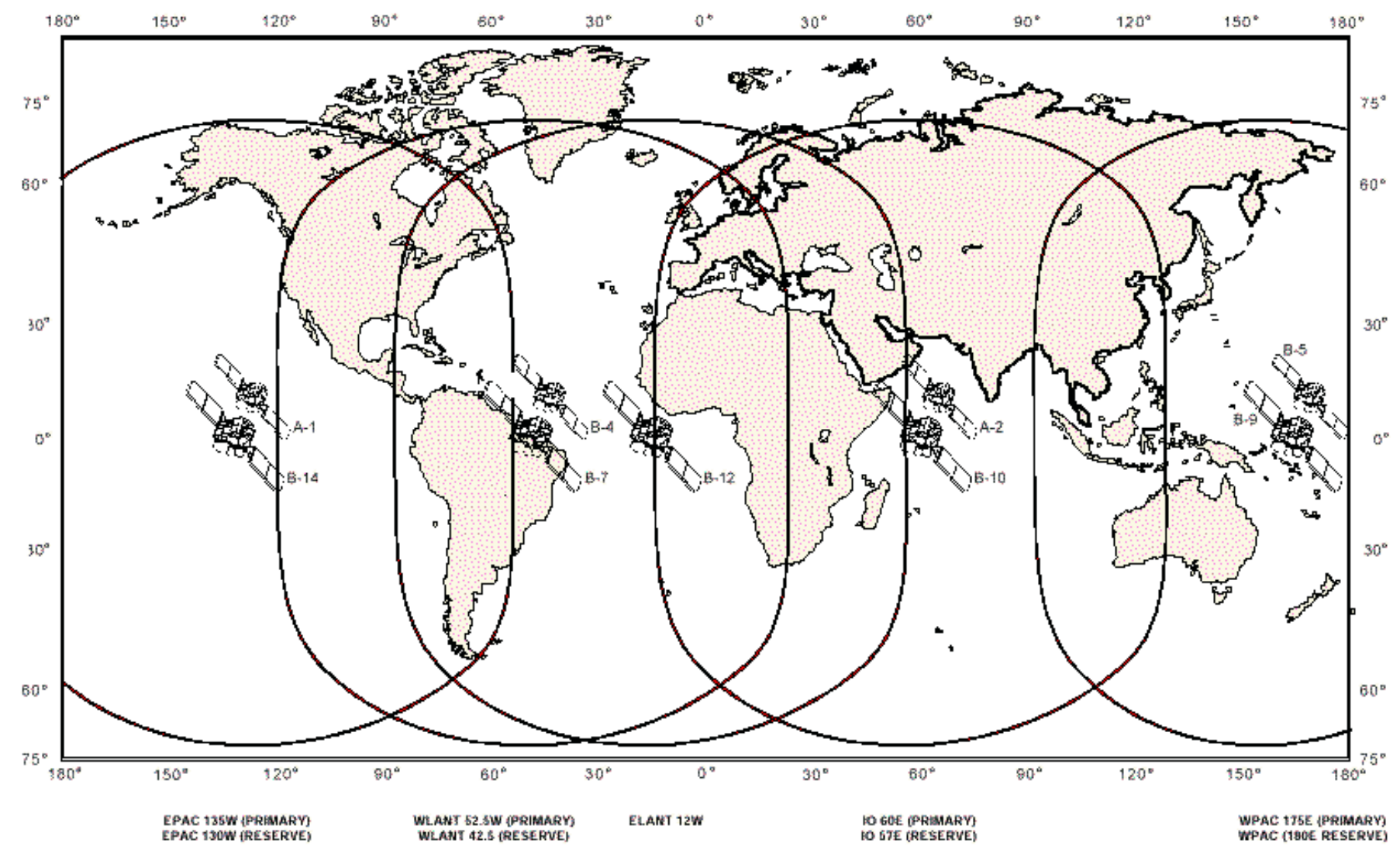




\section{Narrowband Signals}

- Multiple Low bandwidth $(5,25 \mathrm{KHz})$ digital channels.

- Well documented and (supposedly) easy to receive by:

\section{lonospheric Researchers Hobbyists}

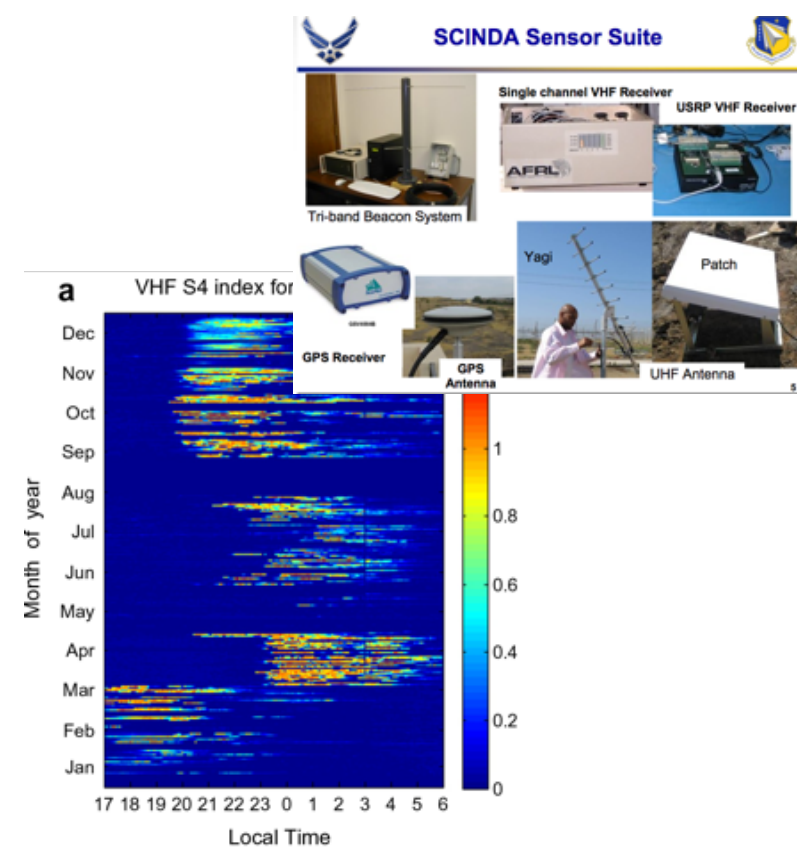

[Olwendo, et al. Adv. Space Research 51 (2013), DOI: . 10.1016/j.asr.2012.12.017]

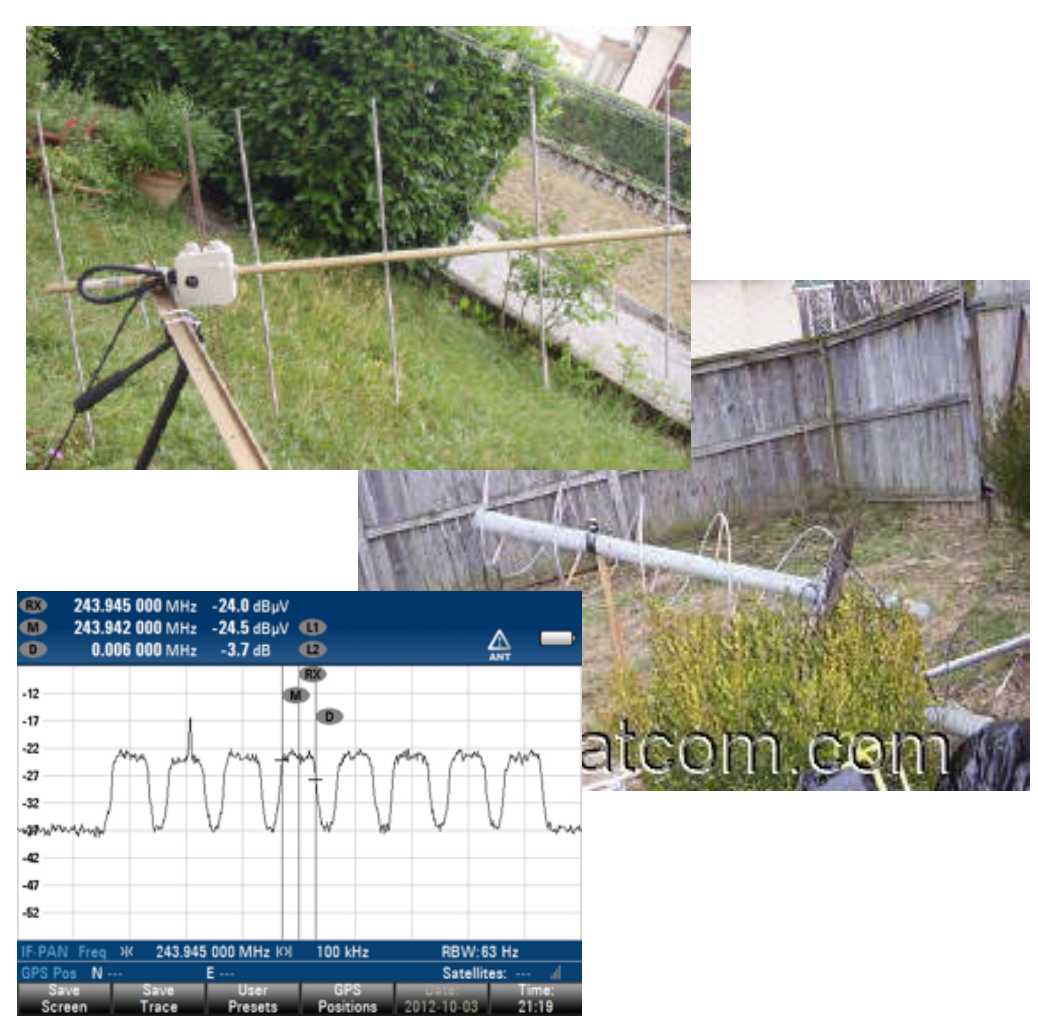

[www.uhf-satcom.com, www.crypto.com]
Pirates

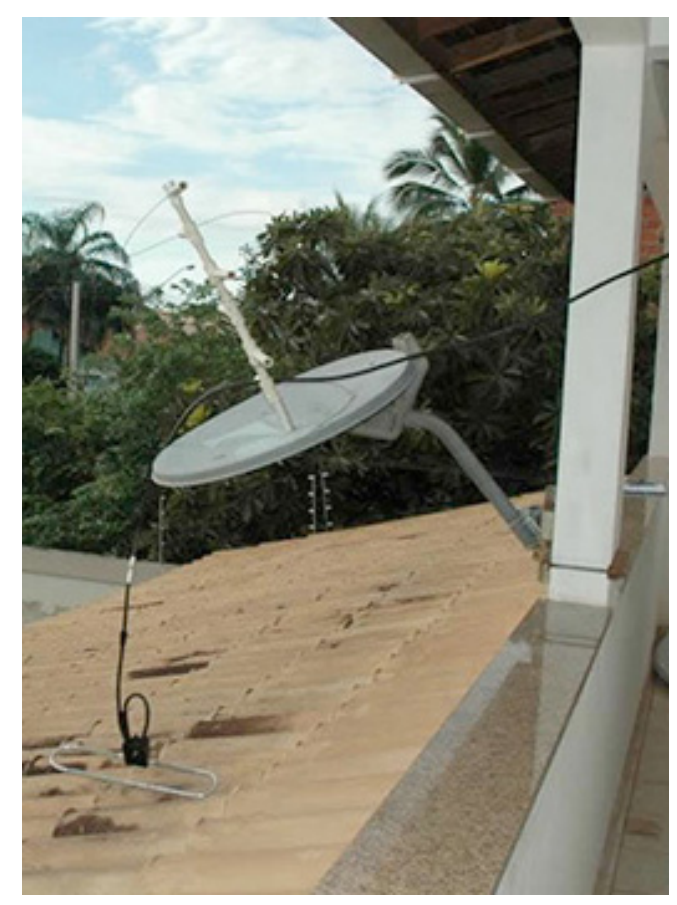

"Nearly illiterate men rigged a radio in less than one minute" [Wired, April 20, 2009] 


\section{P-Band Allocation}

Wide government use:

"The band 225-328.6 MHz is used for a diverse array of land-based, airborne, maritime, and satellite radio communications services by the military forces, National Guard units, Federal Aviation Administration (FAA), Coast Guard (CG), National Aeronautics and Space Administration (NASA), Department of Energy (DOE), and other Federal agencies. Tactical and non-tactical mobile communications, mobilesatellite communications, and air traffic control communications are the most prevalent uses."

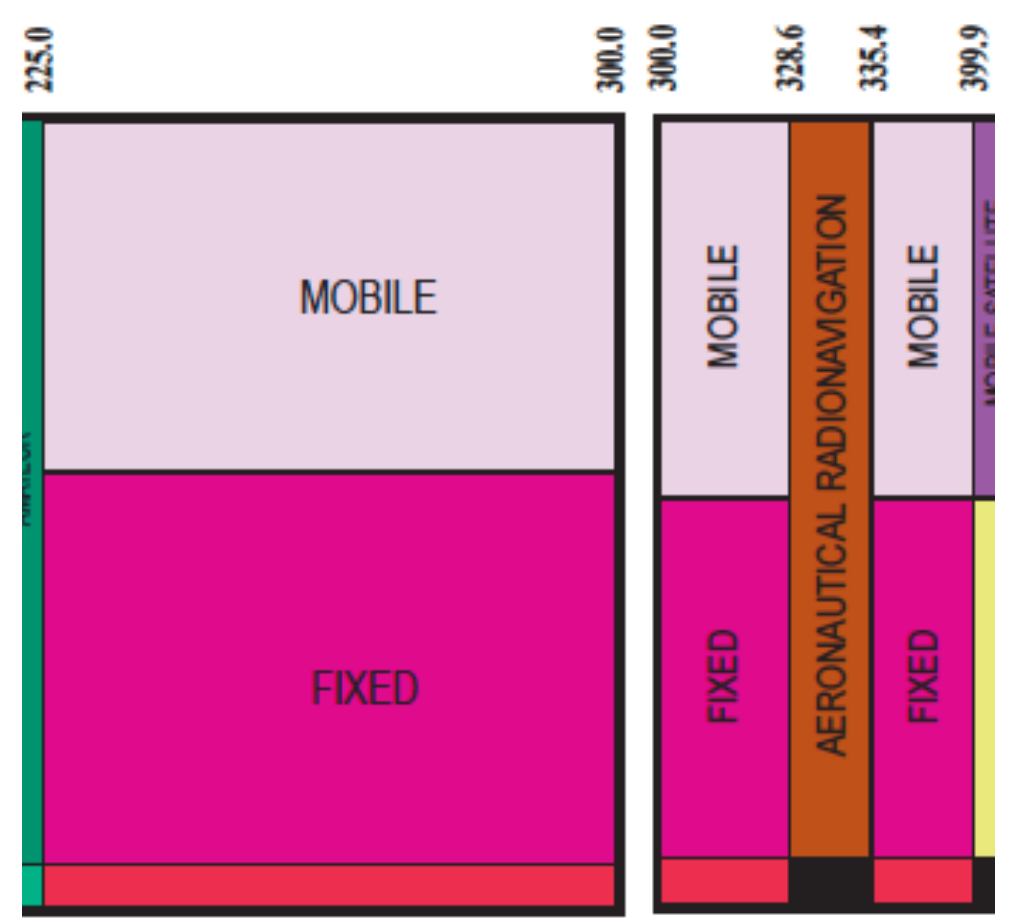

Federal Government Spectrum Compendium, December 16, 2015. National Telecommunications and Information Administration [Online.] https://www.ntia.doc.gov/print/other-publication/2015/federalgovernment-spectrum-compendium 


\section{(19. Gradand Signals of Opportunity Airborne PURDUE Demonstrator (SoOp-AD)}
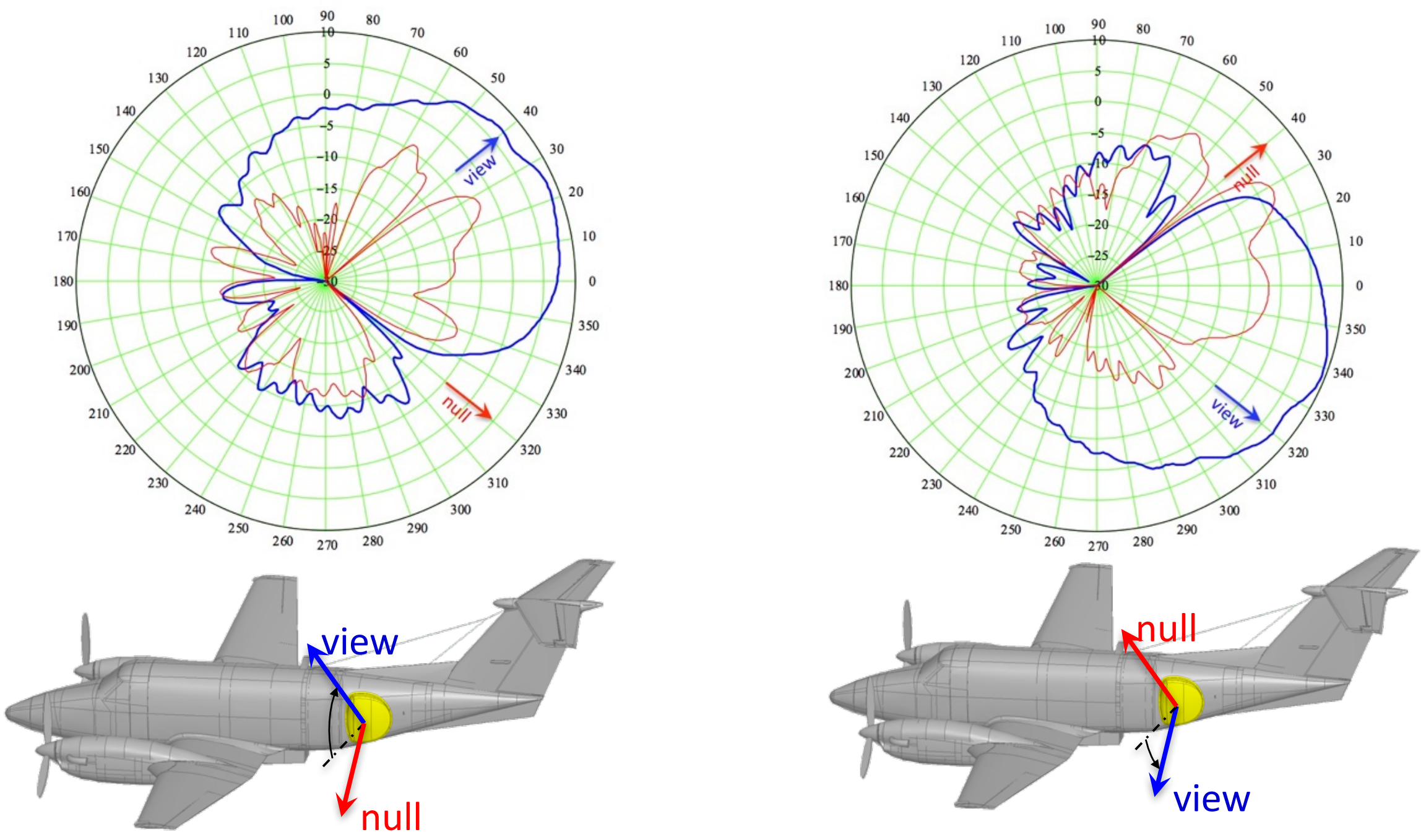


\section{Mrod Godand}

First Look at Data: Antenna Null-Steering and Adjustment I Y Y

- Lake Ellsworth Overflights

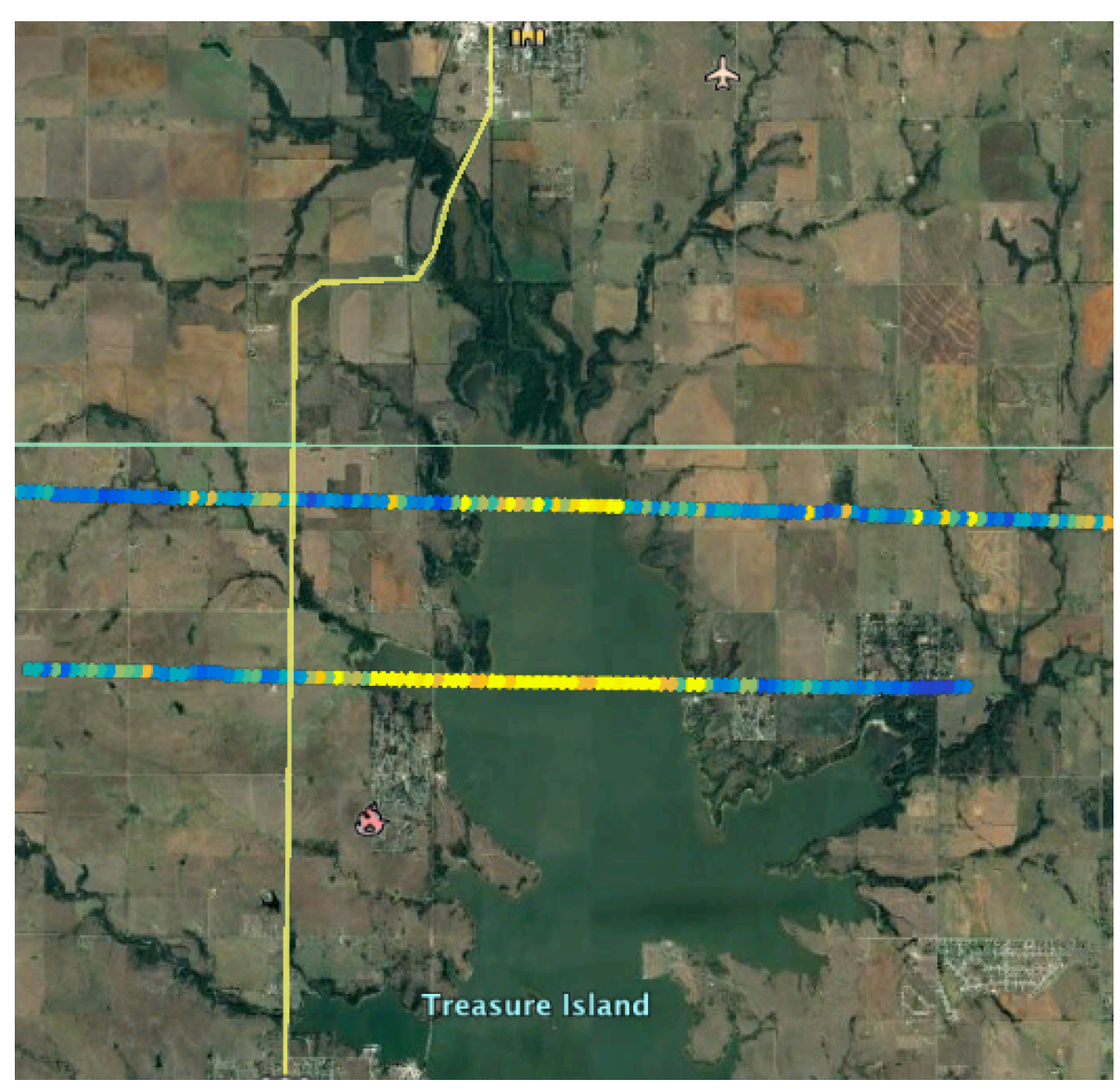

Science Flight 3 (10/22)

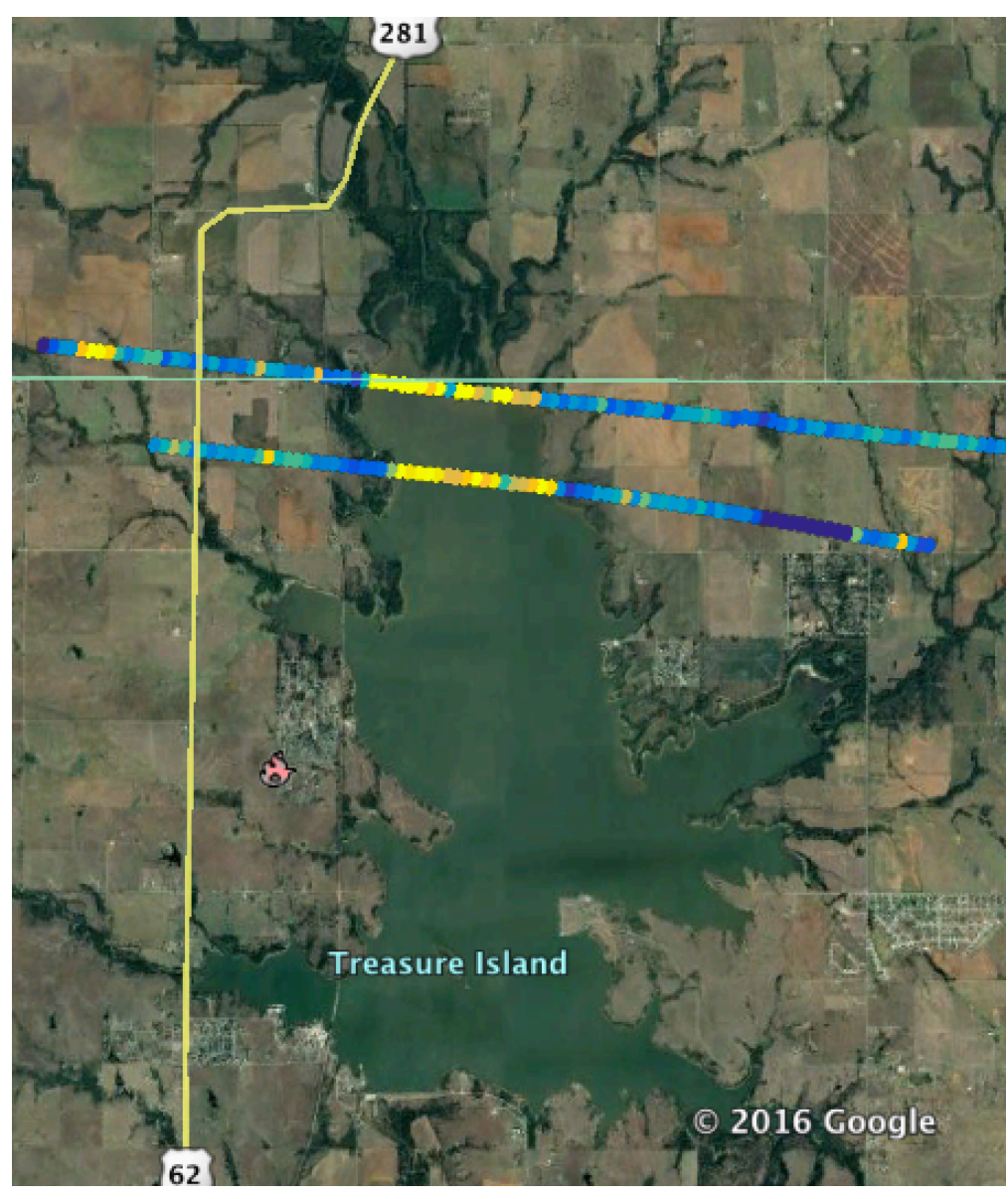

Science Flight 5 (10/25) 


\section{Estimating Reflectivity}

Auto-correlation of channel 1

$$
\begin{aligned}
R_{11}(\tau) & =\left\langle x_{1}^{*}(t) x_{1}(t+\tau)\right\rangle \\
& =G_{1} G_{S, D} C_{D}\left\langle a^{*}\left(t-\tau_{D}\right) a\left(t-\tau_{D}+\tau\right)\right\rangle+G_{1} \sigma_{1}^{2} \delta(\tau)
\end{aligned}
$$

Cross-correlation of channel 1 and 2

$$
\begin{aligned}
R_{12}(\tau) & =\left\langle x_{1}^{*}(t) x_{2}(t+\tau)\right\rangle \\
& \left.=\sqrt{G_{1} G_{2} G_{S, D} G_{E, R} \Gamma} C_{D}\left\langle a^{*}\left(t-\tau_{D}\right) a\left(t-\tau_{R}+\tau\right)\right\rangle e^{j \omega\left(\tau_{R}-\tau_{D}\right.}\right)
\end{aligned}
$$

Reflectivity:

$$
\frac{R_{12}\left(\tau_{R D}\right)}{R_{11}(0)-G_{1} \sigma_{1}^{2}}=\sqrt{\frac{G_{2} G_{E, R}}{G_{1} G_{S, D}}} \sqrt{\Gamma} e^{j \omega \cdot \tau_{R D}}
$$

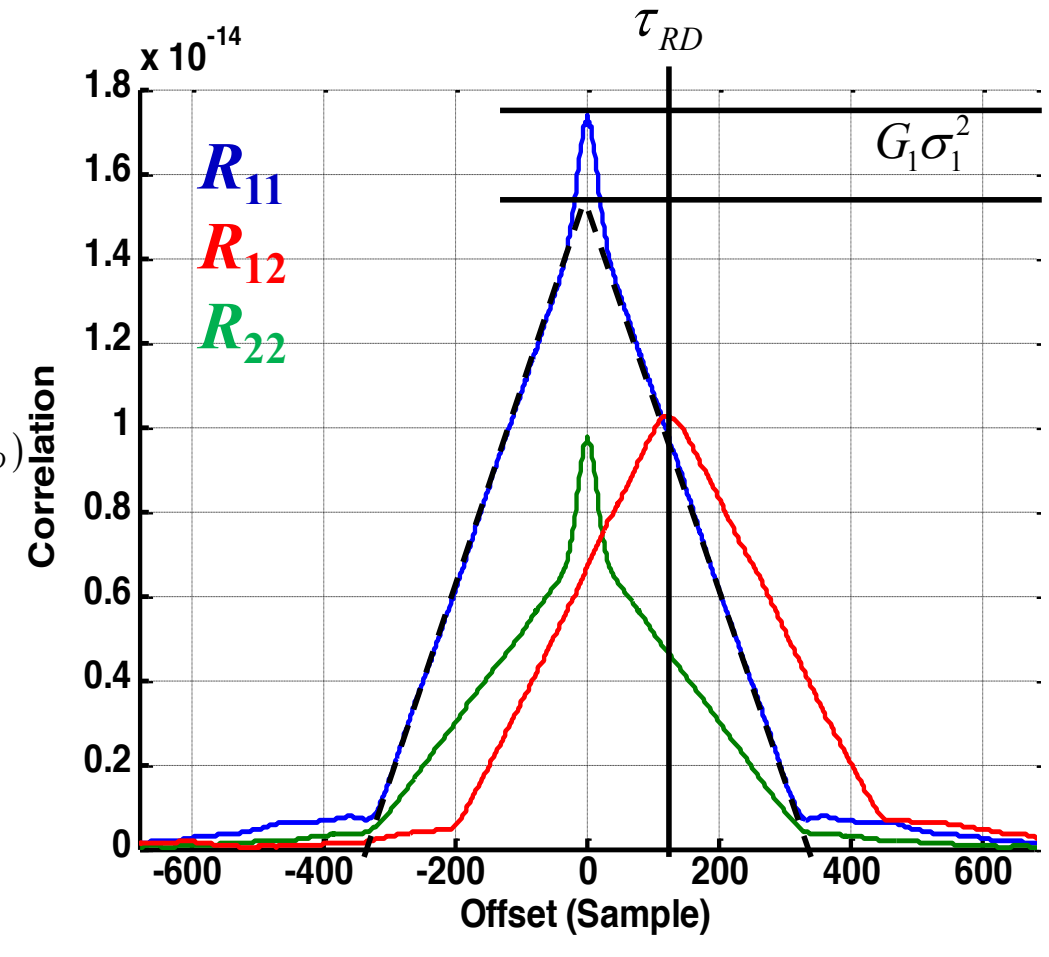

Reflectivity: 0.65, EIRP: $26 \mathrm{~dB}$ 


\section{RFI Impacts Estimate}

- RFI in sky antenna (channel 1) adds to $R_{11}(0)$, which attenuates estimate by:

$$
\sim\left(\frac{1}{1+I S R}\right)^{2} \quad I S R=\frac{I_{D}}{C_{D}}
$$

- RFI in Earth antenna increases noise

- RFI in both antennas adds to $R_{12}$

- Systematic addition or subtraction, else

- Increase noise if $\tau_{R D} \gg 1 / B W_{I}$

Reflectivity:

$$
\frac{R_{12}\left(\tau_{R D}\right)}{R_{11}(0)-G_{1} \sigma_{1}^{2}}=\sqrt{\frac{G_{2} G_{E, R}}{G_{1} G_{S, D}}} \sqrt{\Gamma} e^{j \omega \tau_{R D}}
$$

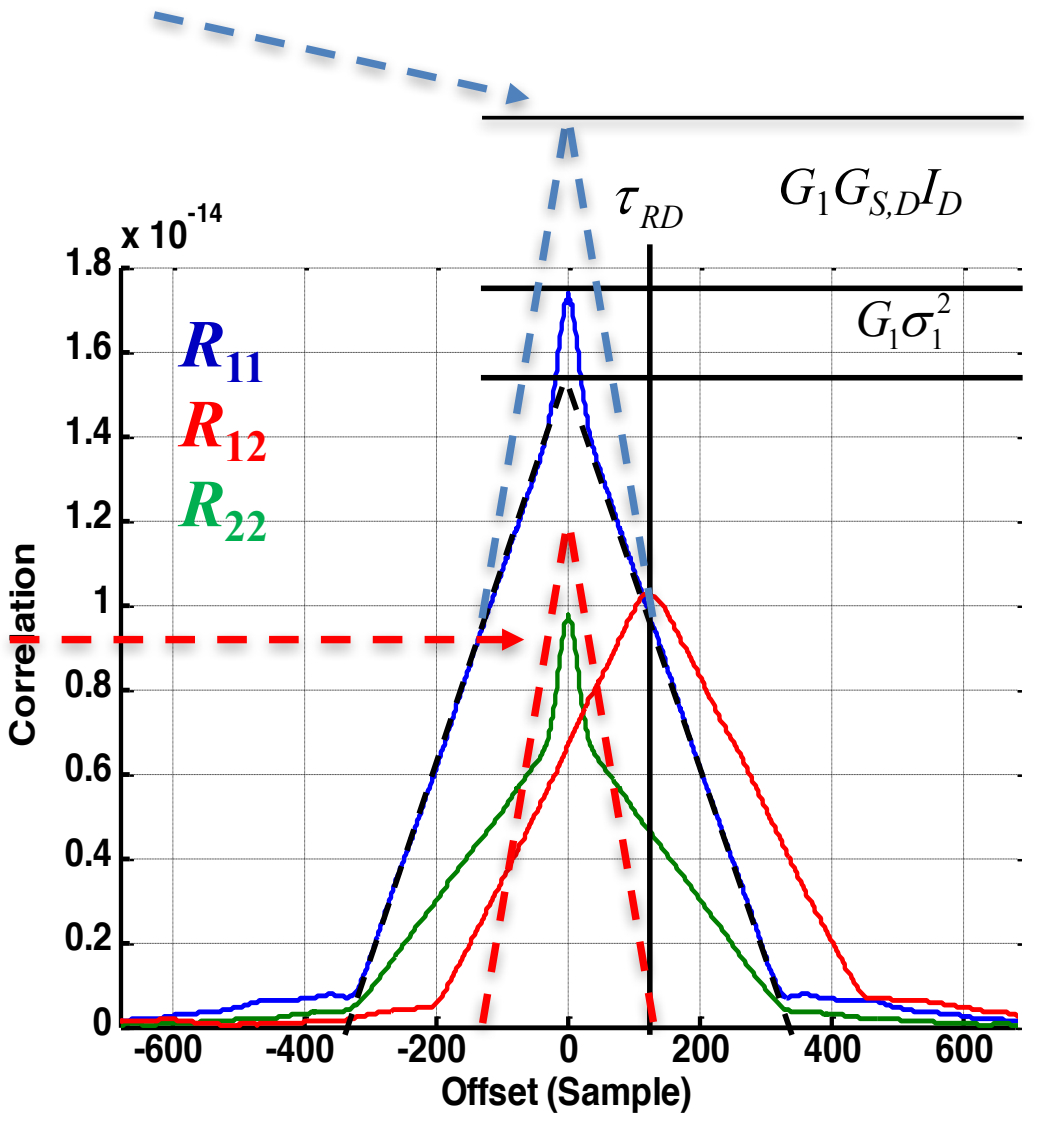

Reflectivity: 0.65, EIRP: $26 \mathrm{~dB}$ 


\section{Urban Local Spectrogram}

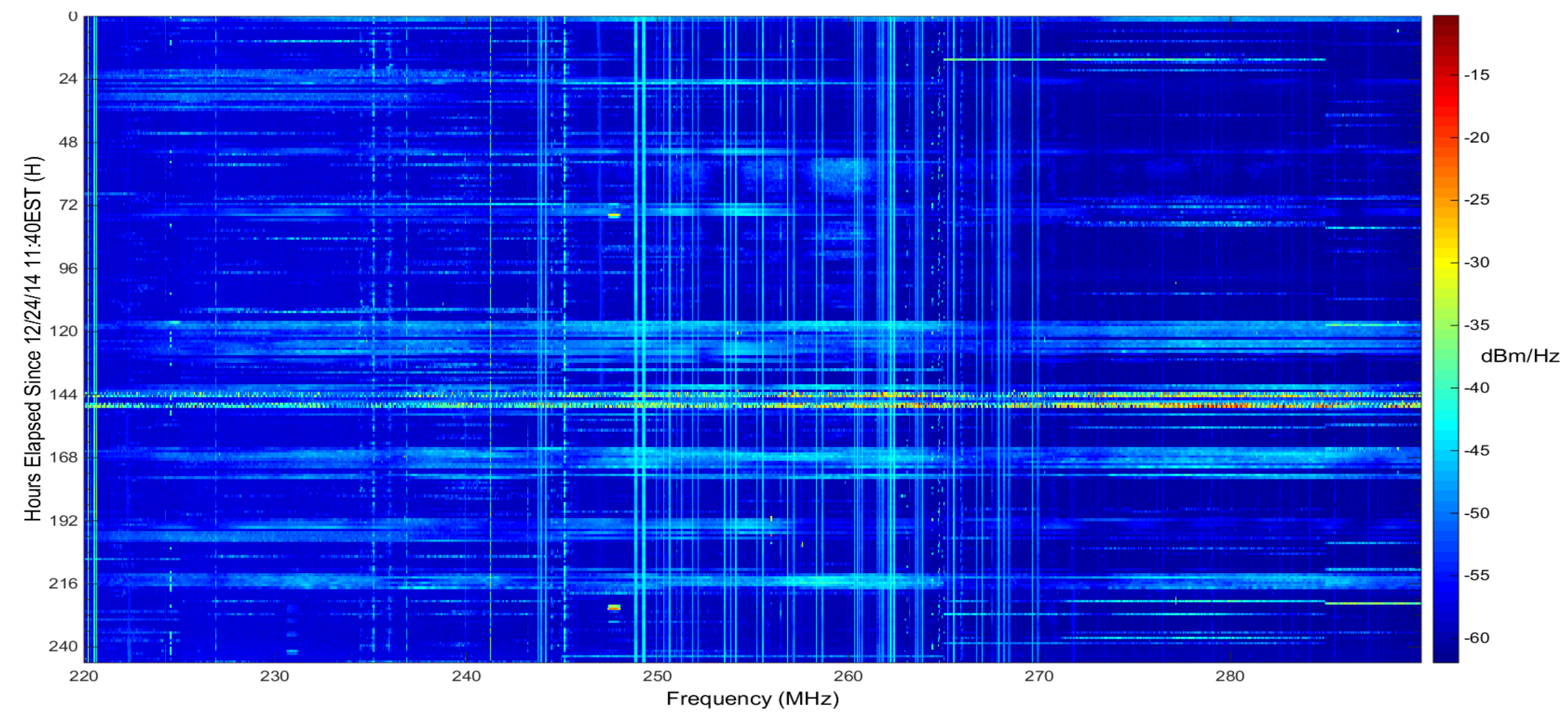

Spectrogram collected near Washington, D.C. shows desired persistent narrowband signals but with significant broadband transient interference. 


\section{Rural Local Spectrum 1}

antenna 1

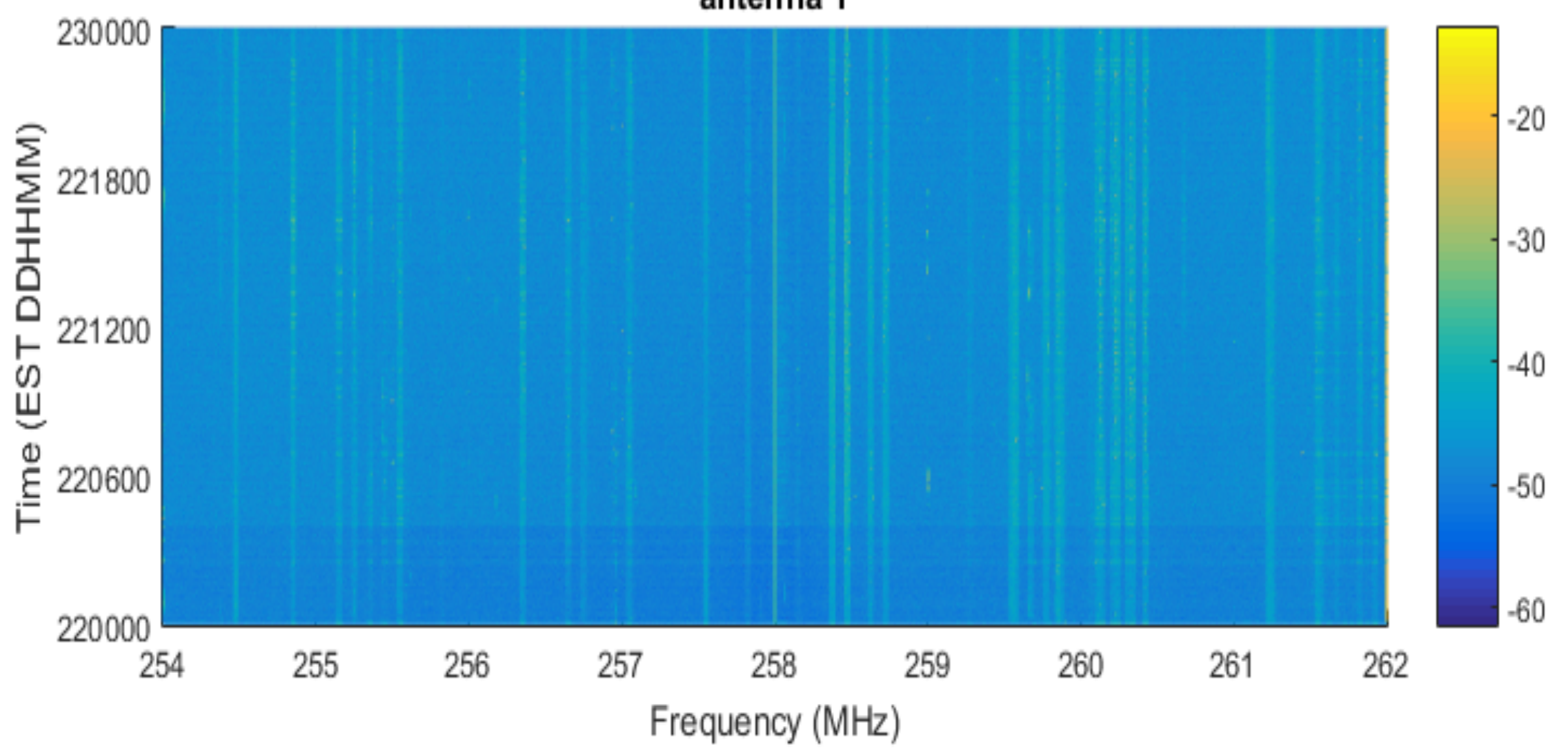

Spectrogram showing desired persistent narrowband signals with no discernable RFI from undesired signals. 


\section{Rural Local Spectrum 2}

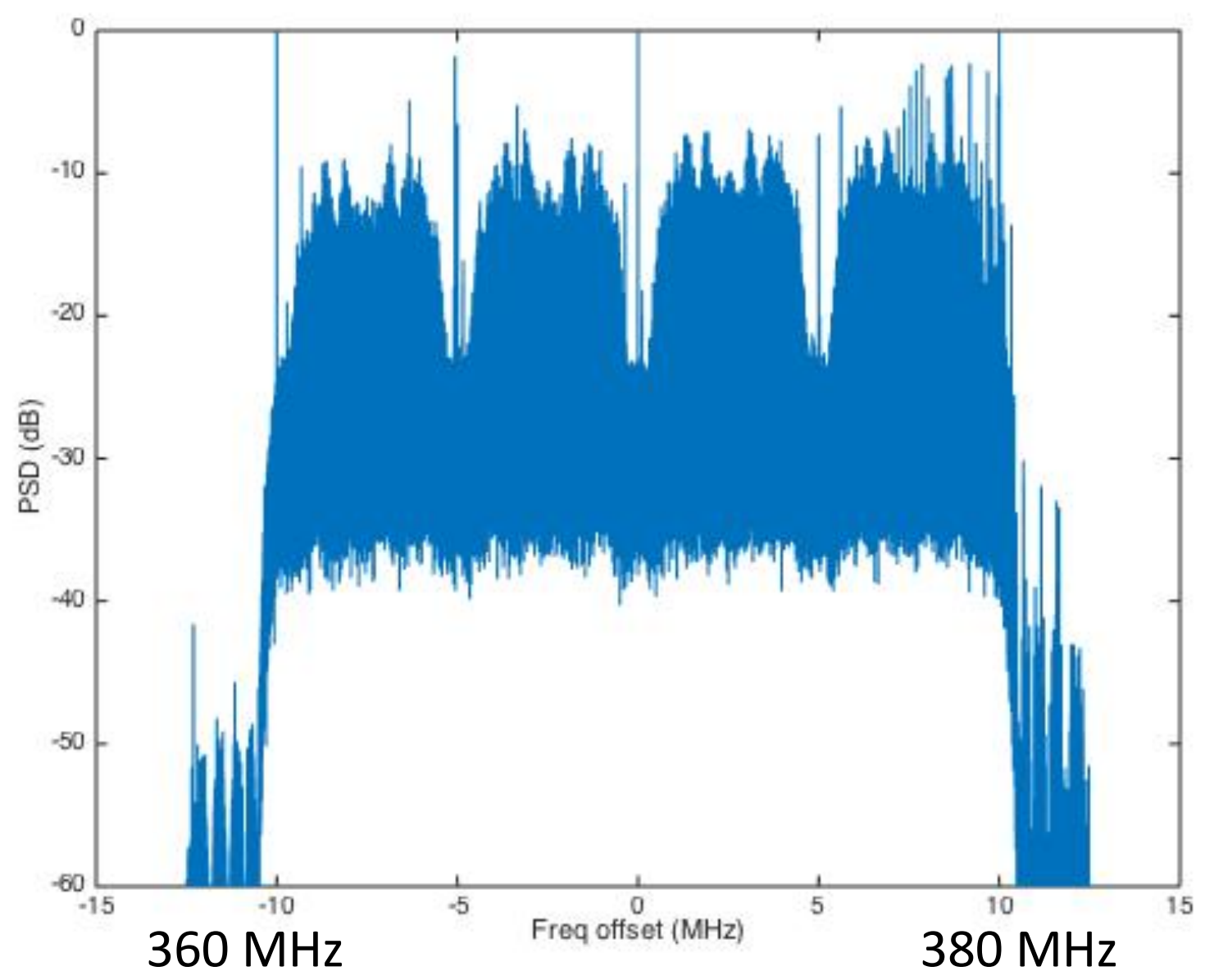

Spectrogram showing desired persistent narrowband signals with potential RFI in band 4 . 


\section{Airborne Spectrum}

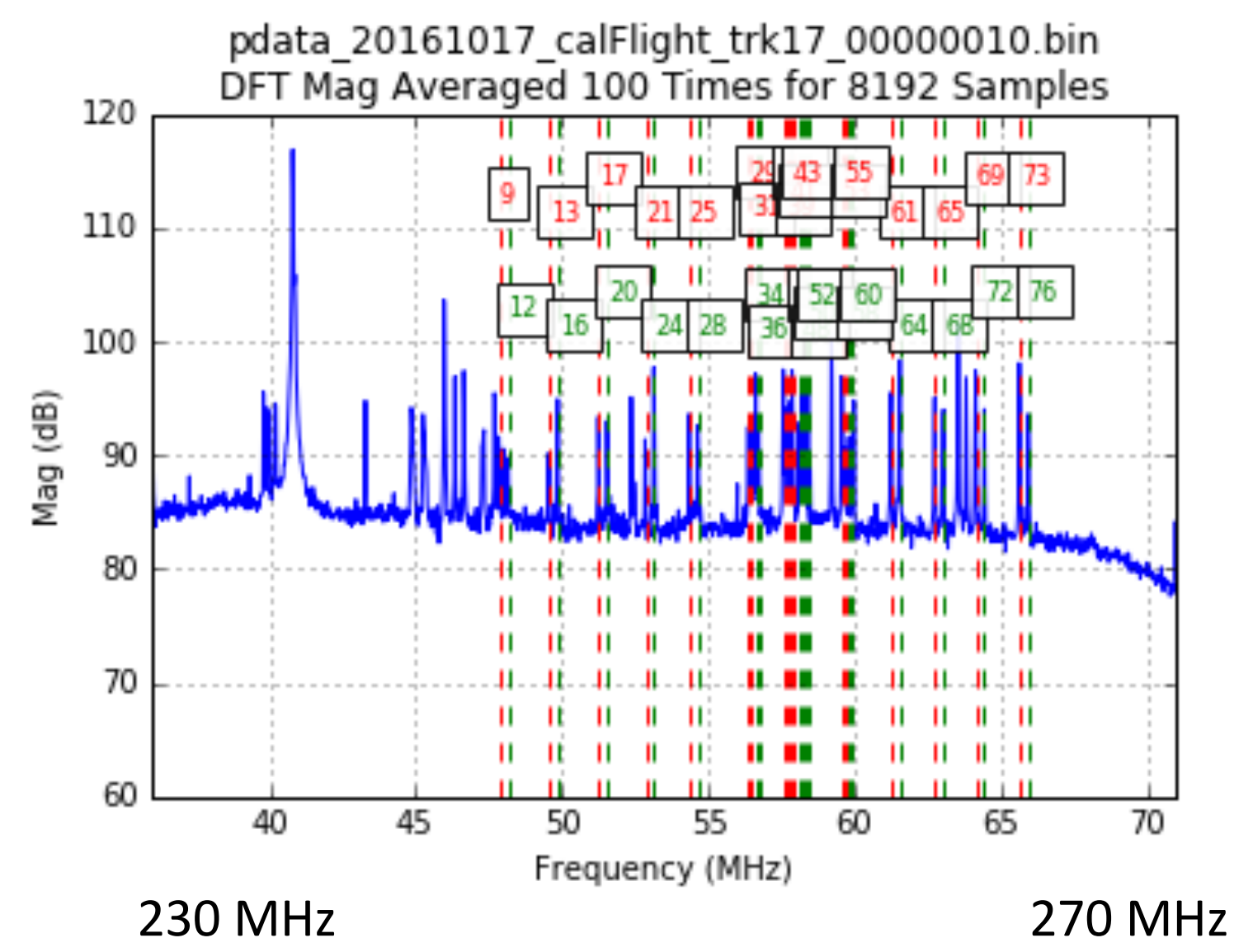

Spectrogram showing desired narrowband signals with no discernable RFI from undesired signals. 


\section{Acknowledgements}

- This work was funded under NASA Grant NNX14AE80G (2013 Instrument Incubator Program).

- USDA (Michael Cosh) provided valuable assistance with utilizing the Little Washita ARS Micronet data.

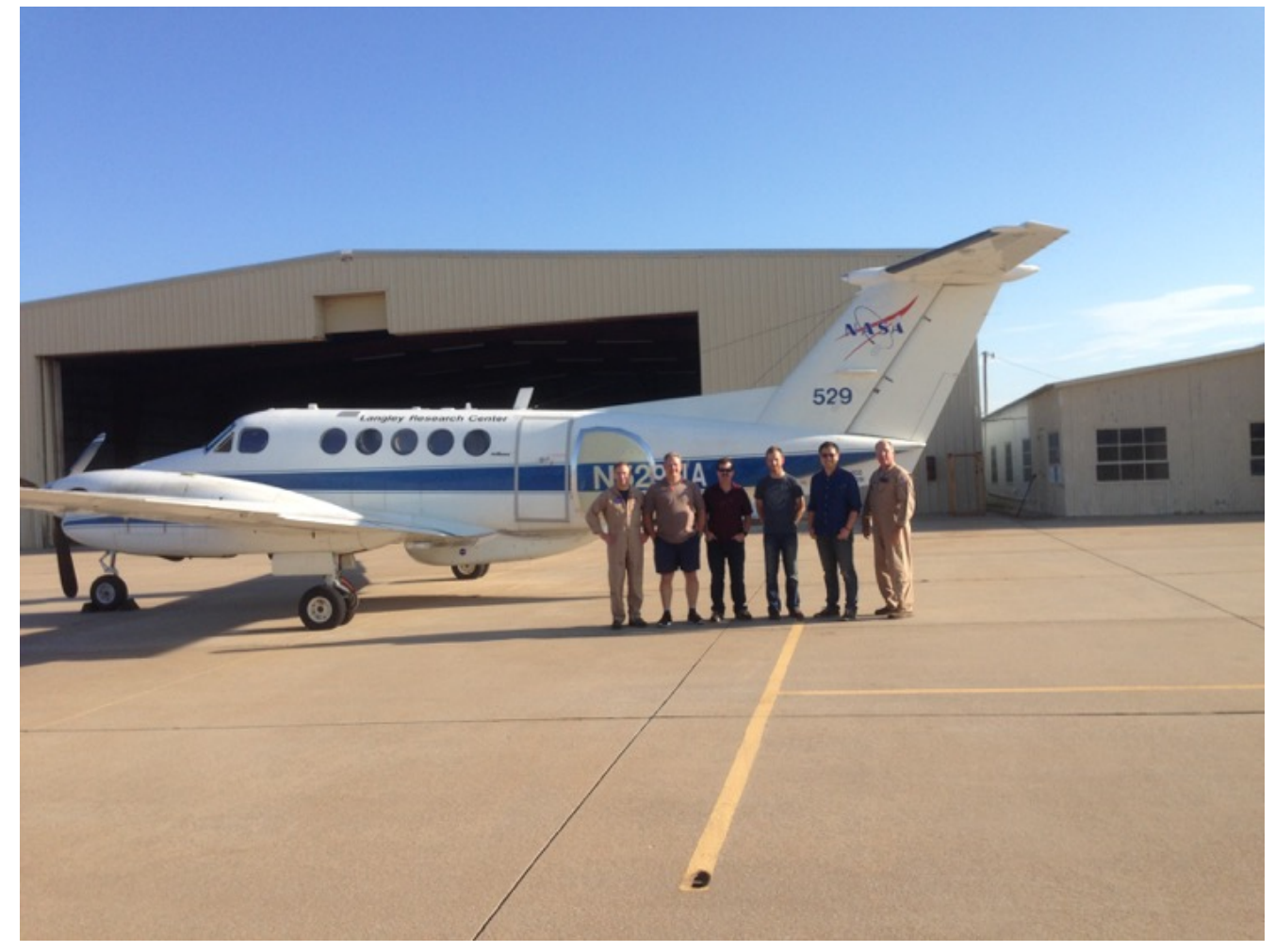




\section{Discussion}

- Rural spectrum cleaner than Urban spectrum

- Urban spectrum possibly worst case because D.C. area

- Airborne spectrum example appears clean 